\title{
3D Kinematic Analysis for the Functional Evaluation in the Rat Model of Sciatic Nerve Crush Injury
}

\section{$\operatorname{AUTHOR}(\mathrm{S}):$}

Wang, Tianshu; Ito, Akira; Tajino, Junichi; Kuroki, Hiroshi; Aoyama, Tomoki

\section{CITATION:}

Wang, Tianshu ... [et al]. 3D Kinematic Analysis for the Functional Evaluation in the Rat Model of Sciatic Nerve Crush Injury. Journal of visualized experiments : JoVE 2020, 156: e60267.

ISSUE DATE:

2020-02

URL:

http://hdl.handle.net/2433/252815

RIGHT:

Copyright (c) 2020 Journal of Visualized Experiments 


\title{
Video Article \\ 3D Kinematic Analysis for the Functional Evaluation in the Rat Model of Sciatic Nerve Crush Injury
}

\author{
Tianshu Wang ${ }^{1}$, Akira Ito ${ }^{2}$, Junichi Tajino ${ }^{1,3}$, Hiroshi Kuroki ${ }^{2}$, Tomoki Aoyama ${ }^{1}$ \\ ${ }^{1}$ Department of Development and Rehabilitation of Motor Function, Human Health Sciences, Graduate School of Medicine, Kyoto University \\ ${ }^{2}$ Department of Motor Function Analysis, Human Health Sciences, Graduate School of Medicine, Kyoto University \\ ${ }^{3}$ Department of Otolaryngology, The Ohio State University Wexner Medical Center
}

Correspondence to: Akira Ito at ito.akira.4m@kyoto-u.ac.jp

URL: https://www.jove.com/video/60267

DOI: doi:10.3791/60267

Keywords: Neuroscience, Issue 156, rat, sciatic nerve injury, three-dimensional, kinematic analysis, functional evaluation, center of gravity

Date Published: 2/12/2020

Citation: Wang, T., Ito, A., Tajino, J., Kuroki, H., Aoyama, T. 3D Kinematic Analysis for the Functional Evaluation in the Rat Model of Sciatic Nerve Crush Injury. J. Vis. Exp. (156), e60267, doi:10.3791/60267 (2020).

\section{Abstract}

Compared to the Sciatic Functional Index (SFI), kinematic analysis is a more reliable and sensitive method for performing functional evaluations of sciatic nerve injury rodent models. In this protocol, we describe a novel kinematic analysis method that uses a three-dimensional (3D) motion capture apparatus for functional evaluations using a rat sciatic nerve crush injury model. First, the rat is familiarized with treadmill walking. Markers are then attached to the designated bone landmarks and the rat is made to walk on the treadmill at the desired speed. Meanwhile, the posterior limb movements of the rat are recorded using four cameras. Depending on the software used, marker tracings are created using both automatic and manual modes and the desired data are produced after subtle adjustments. This method of kinematic analysis, which uses a 3D motion capture apparatus, offers numerous advantages, including superior precision and accuracy. Many more parameters can be investigated during the comprehensive functional evaluations. This method has several shortcomings that require consideration: The system is expensive, can be complicated to operate, and may produce data deviations due to skin shifting. Nevertheless, kinematic analysis using a 3D motion capture apparatus is useful for performing functional anterior and posterior limb evaluations. In the future, this method may become increasingly useful for generating accurate assessments of various traumas and diseases.

\section{Video Link}

The video component of this article can be found at https://www.jove.com/video/60267/

\section{Introduction}

The Sciatic Functional Index (SFI) is the benchmark method for carrying out functional sciatic nerve evaluations ${ }^{1}$. The SFI has been widely adopted and is frequently used within various functional evaluation studies on rat sciatic nerve injuries ${ }^{2,3,4,5,6}$. In spite of its popularity, there are several problems with SFI, including automutilation ${ }^{7}$, joint contracture risk, and smearing of the footprints ${ }^{8}$. These problems seriously affect its prognostic value ${ }^{9}$. Therefore, an alternative, less error-prone method is required as a substitute for the SFI.

One such alternative method is kinematic analysis. This includes comprehensive gait analysis using tracking markers attached to bony landmarks or joints. Kinematic analysis is increasingly used for functional evaluations ${ }^{9}$. This method is progressively being recognized as a reliable and sensitive tool for functional evaluation ${ }^{10}$ without the shortcomings attributed to the SFI ${ }^{11,12}$.

In this protocol, we describe a series of kinematic analyses that use a 3D motion capture apparatus consisting of a treadmill, four $120 \mathrm{~Hz}$ charged coupled device (CCD) cameras, and data processing software (see Table of Materials). This kinematic analysis method differs from general video walking or gait analysis ${ }^{13,14}$. Two cameras are positioned in different directions to record posterior limb movements from a single side. Subsequently, a 3D digital model of the posterior limb is constructed using computer graphics ${ }^{9}$. We can calculate designated joint angles, such as hip, knee, ankle, and toe joint, by closely recapitulating the actual limb dimensions. Additionally, we can determine various parameters such as stride/step length and the ratio of the stance phase to the swing phase. These reconstructions are based on a completely reconstructed 3D digital model of the posterior limbs, generated from data transmitted by two sets of cameras. Even the imaginary center of gravity (CoG) trajectory can be calculated automatically.

We used this 3D motion capture apparatus to introduce and assess multiple kinematic parameters that reveal functional changes over time within the context of the rat sciatic nerve crush injury model. 


\section{Protocol}

The protocol was approved by the animal experimentation committee of Kyoto University, and all protocol steps were performed in accordance with the Guidelines of the Animal Experimentation Committee, Kyoto University (approval number: MedKyo17029).

\section{Familiarizing rats with treadmill walking}

1. Set up two transparent plastic sheets on both sides of the treadmill to let a 12-week-old male Lewis rat walk in a straight, frontward direction, then turn on the electric shock grid.

2. Have each rat walk on the treadmill. Gradually accelerate the treadmill to the desired speed $(20 \mathrm{~cm} / \mathrm{s}$ or $12 \mathrm{~m} / \mathrm{min})$ and let the rat walk normally at this speed for $5 \mathrm{~min}$. After each walking session, provide a 1-2 min rest break. Repeat this process $3 x$ per day, 5 days per week, for 1 week.

NOTE: Start the treadmill walking 1 week before step 2 .

3. House rats in groups of three per cage with a $12 \mathrm{~h}$ light-dark cycle and feed them commercial rat food and tap water ad libitum.

\section{Performing the sciatic nerve crush injury}

1. Place the rat in an anesthesia induction chamber and introduce $5 \%$ isoflurane inhalation solution.

2. Provide an intraperitoneal injection of a combination anesthetic prepared with $0.15 \mathrm{mg} / \mathrm{kg}$ medetomidine hydrochloride, $2 \mathrm{mg} / \mathrm{kg}$ midazolam, and $2.5 \mathrm{mg} / \mathrm{kg}$ butorphanol tartrate to the rat. Check for the lack of pedal reflexes. Then shave an area from the left greater trochanter to the mid-thigh with an electric shaver.

3. Spread out a piece of aseptic cloth, place the rat on it, and have it lie in the left lateral position. Place sterile surgical instruments on the cloth as well.

4. Create a straight incision from the greater trochanter to the mid-thigh with a surgical no. 10 blade. Then perform a blunt dissection between quadriceps femoris and biceps femoris using a surgical hemostat to expose the sciatic nerve.

5. Detach the sciatic nerve from the surrounding tissue with two pairs of microforceps and crush the sciatic nerve for $10 \mathrm{~s}$, using a standard surgical hemostat, to create a $2 \mathrm{~mm}$ long crush injury at the site directly below the gluteal tuberosity.

6. Perform a 9-0 nylon epineural stitch at the proximal end of the injury using a pair of microforceps and then close the muscle and skin with 4-0 nylon sutures.

7. Provide an intraperitoneal injection of an anesthetic antagonist prepared with $0.3 \mathrm{mg} / \mathrm{kg}$ atipamezole hydrochloride to the rat, to wake it up within $10 \mathrm{~min}$. After the rat recovers from anesthesia, observe the left toe movements while the rat is suspended by the base of its tail. If the toe does not spread at all, the surgery was successful.

8. House the rats individually after the surgery with a $12 \mathrm{~h}$ light-dark cycle and feed them commercial rat food and tap water ad libitum.

\section{Attaching the markers}

1. Place the trained rat in an anesthesia induction chamber and introduce a $5 \%$ isoflurane inhalation solution. Check for the lack of the pedal reflex by pinching the toe.

2. Allow the rat to be continuously anesthetized using an anesthetic mask ( $2 \%$ isoflurane inhalation solution). While the rat receives stable anesthesia, shave an area from the lower back to the bilateral malleoli using an electric shaver.

CAUTION: To prevent exposing researchers to the leaking isoflurane, make sure that the mask tightly covers the head and face of the rat. NOTE: To prevent injury to the rat, shave the hair off as gently as possible.

3. Place the rat in the prone position. Use a black marker pen to mark the following bone landmarks on the shaved skin: A line through the spinous processes from the lumbar to sacral vertebrae, the anterior superior iliac spines, the greater trochanters, the knee joints, the lateral malleoli, the fifth metatarsophalangeal joints, and the tip of the fourth toe.

NOTE: The line through the spinous processes is used for determining whether the bilateral markers are axially symmetric.

4. Use a liquid adhesive to attach hemispheric markers to these bone landmarks, except for the line through the spinous processes from the lumbar to the sacral vertebrae, and the tip of the fourth toe. Use distinct colors for every other landmark to avoid confusion. The tip of the fourth toe is marked with pink ink.

CAUTION: Take care not to drip adhesive onto the exposed skin of the operator.

5. After placing all the markers, put the rat back into the cage. Do not put the rat on the treadmill until it fully recovers from anesthesia. NOTE: Reduced consciousness may seriously influence normal walking if the rat does not fully recover from anesthesia.

\section{Calibration and software setup}

1. Set up two transparent plastic sheets on both sides of the treadmill and place the calibration box in the middle of the treadmill. Open the recording software and then click on the Calibration Image icon on the display (Supplementary File 1).

2. Click the Recording icon to record $1-2 \mathrm{~s}$ of video from four directions using $120 \mathrm{~Hz}$ CCD cameras. Click on the Recording icon again to stop the recording. NOTE: The video will be saved automatically once the recording stops.

3. Open the video file in the calculation software. Click and drag the characteristic points of the calibration box 3D models on the bottom right corner of the screen to the corresponding markers on the four pictures, which are automatically transformed from the video in the calibration pattern (Supplementary File 2). Then click on the Save icon. NOTE: Do not change the positions of the cameras after the calibration is completed. 


\section{Recording the walking}

1. Take the calibration box out of the treadmill, turn on the electric shock grid, and place the fully awake rat on the treadmill. Open the recording software and input the basic information about the rat, including its serial number, walking speed, and the name of the principal operator.

2. Turn on the treadmill and set the speed to $20 \mathrm{~cm} / \mathrm{s}$. After the rat adapts to the speed and is able to walk normally, click the Recording icon on the display to record the walking rat with the four cameras. Once enough steps are recorded $(>10)$, click on the icon again to stop recording, and turn off the treadmill.

NOTE: The video will be saved automatically once the recording stops.

3. Put the rat back into the anesthesia induction chamber for anesthetization. While the rat is under continuous anesthesia (administered via the anesthetic mask), remove the hemispheric markers. NOTE: Remove the markers as gently as possible to avoid causing pain to the rat.

4. At the designated time (e.g., 1 week, 3 weeks, or 6 weeks after surgery), perform the kinematic measurement on the rat by repeating steps 3.1-5.3. Make the kinematic measurement only once, at the beginning of the experiment, for the rats that did not receive surgery (i.e., the control group).

\section{Marker tracing}

1. Open the calculation software and open the video file on the interface.

2. Click and drag the bilateral control bar on the progress bar of the video to ensure that only a 10-step treadmill walking record is displayed (Supplementary File 3). Click and drag each characteristic point from the 3D model on the bottom right corner of the screen to the corresponding marker on each of the four initial pictures of the videos that were taken by the cameras (Supplementary File 4).

3. Click on the Automatically Tracing icon to start the automatic marker tracing process (Supplementary File 5, Supplementary File 6). If the system does not accurately trace a marker, click on the Manually Digitize icon to switch to the manual tracing mode (Supplementary File 7 ), click on the tracing characteristic point in the $3 \mathrm{D}$ model, and then on the responding marker in the picture.

4. Once the marker is clicked, ensure that the picture switches to the next frame of the video. Now continuously click on the marker until the marker tracing process is completed. Once finished, click on the Save icon.

\section{Kinematic analysis}

1. Open the analysis software and then open the processed video file on the interface.

2. Click on the Setting icon and select and add designated parameters such as the ankle angle, toe angle, and pelvic shift (X and $Z$ axes) to the Display List in the pop-up window on the right (Supplementary File 8). Click on OK, so that curves representing the value changes in the parameters appear on the interface.

3. Click on the Measurement icon and select Smooth processing in its pull-down menu. Enter $20 \mathrm{~Hz}$ in the pop-up window to remove frequencies greater than $20 \mathrm{~Hz}$ within the curves (Supplementary File 9).

4. Ensure that there are five panels on the interface: the walking video of the rat, the dynamic 3D model, curves that represent value changes in the parameters from the 10-step cycle, curves that represent mean value changes in parameters, and histograms and schematic diagrams that represent the ratio of the stance and swing phase (Supplementary File 10).

5. Right click the panel for curves representing mean value changes in parameters and select Data Output in the pull-down menu (Supplementary File 11). This will produce the average values of the posterior limb joint angles, including the ankle and toe angles, pelvic shift, and any other desired parameters in 10-step cycle periods.

\section{Representative Results}

We selected four parameters to investigate functional changes over time in a rat sciatic nerve crush injury model. These were the ratio of the stance-to-swing phase, center of gravity (CoG) trajectory, ankle angles, and toe angles in the 'toe off' phase ${ }^{9}$. Twenty-four rats were randomly assigned to one of four groups: the control group (C), rats at the first (1w), third (3w), and sixth (6w) week following left sciatic nerve crush injury.

By means of 3D kinematic analysis, the mean ratio of the stance or swing phase in the 10-step cycle was automatically calculated and represented on the interface (Figure 1A-D). We found that the ratio of the stance-to-swing phase was recovered following surgery.

The CoG is a virtual point that can be traced with a virtual marker by the 3D motion capture apparatus. It is located at the cross point of two lines connecting either of the two anterior superior iliac spines to their contralateral greater trochanters. Thus, the real time pelvic shift in the coronal plane ( $X$ and $Z$ axes) results in a simultaneous shift of the CoG as a 3D constructed pelvic model is used. This shift can also be automatically measured. The CoG trajectory is described as the changing curve of the pelvic shift mean value in the $X$ and $Z$ axes of the 10 -step cycle. The normal CoG trajectory shape resembles the infinity sign $(\infty)$. We found that the CoG trajectory shape did not return to an approximately normal shape until 6 weeks after surgery (Figure 2A-D).

The normal ankle and toe angles in the "toe off" phase reach maximum value during the terminal stance of the step cycle ${ }^{15}$, but these parameters might be falsely reported if the rat has received surgery. Nevertheless, the 3D kinematic analysis allowed us to determine the angles in the "toe off" phase by referring to the video. The mean value of the ankle or toe angle in the "toe off" phase was calculated from the 10-step cycle. The results suggested that the ankle and toe angles, in the "toe off" phase improved in an upward direction after surgery. (Figure $3 \mathbf{A}-\mathbf{B}$ ). 


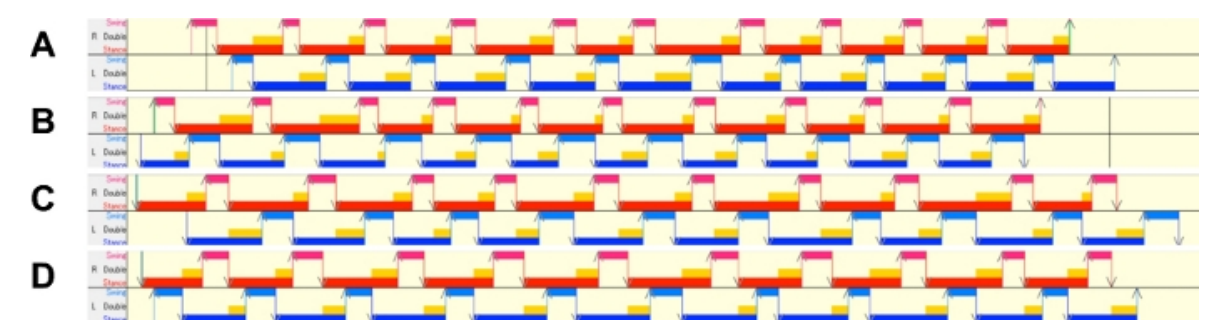

Figure 1: Bilateral stance and swing phase. The right swing (magenta), right stance (red), left swing (azure), and left stance (blue) phases are represented by their respectively colored bars. Yellow bars symbolize double support phases. Panels A-D show each bilateral stance and swing phase in 10-step cycle periods for the control group (A), 1w (B), 3w (C), and 6w (D) groups. C = control; $1 \mathrm{w}=1 \mathrm{week}$ post-surgery; $3 \mathrm{w}=3$ weeks post-surgery; $6 \mathrm{w}=6$ weeks post-surgery. Please click here to view a larger version of this figure.
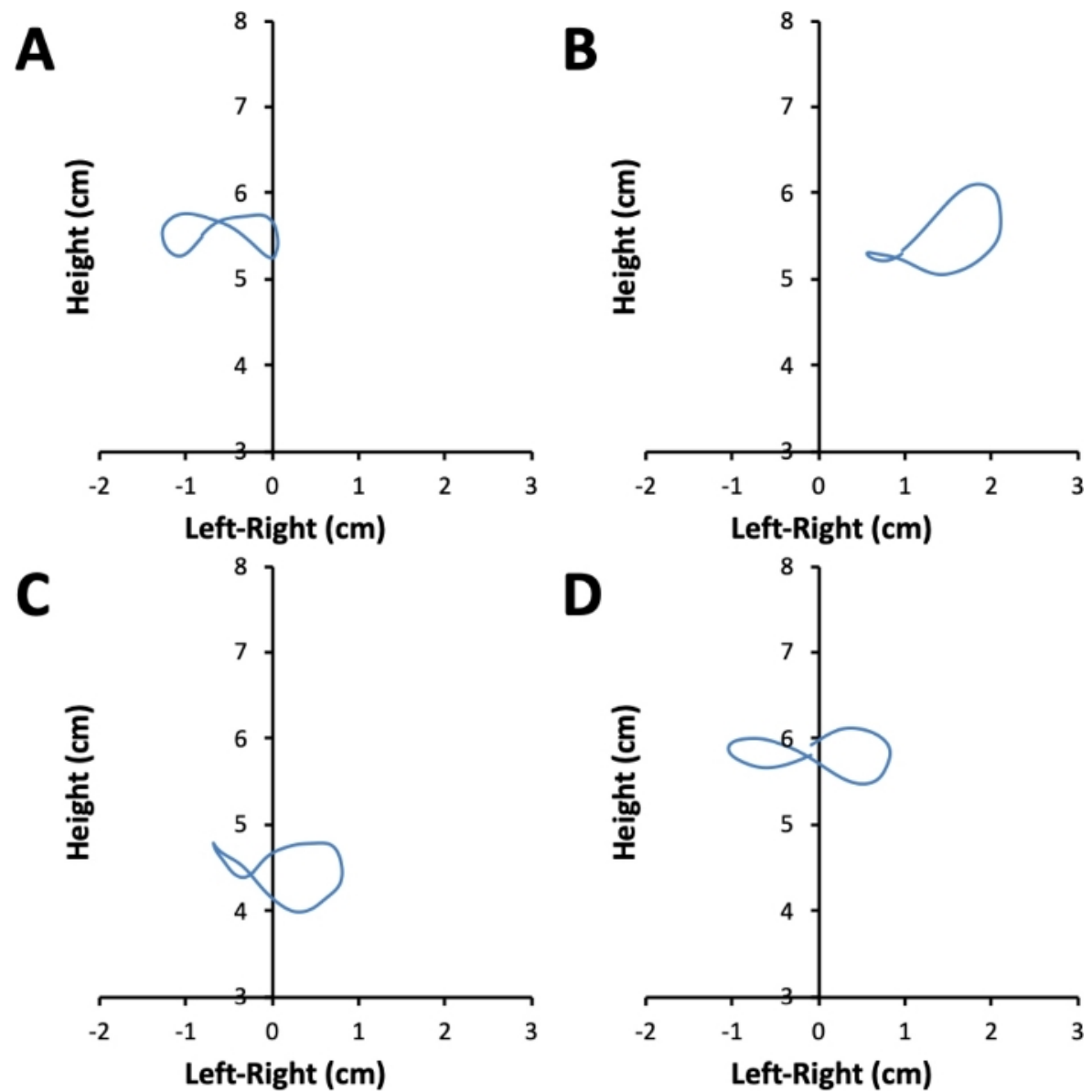

Figure 2: CoG trajectories. Panels A-D show representative average CoG trajectories during 10-step cycle periods for the control group (A), $1 \mathrm{~W}$ (B), $3 w(C)$, and $6 w(D)$ groups. $C=$ control; $1 w=1$ week post-surgery; $3 w=3$ weeks post-surgery; $6 w=6$ weeks post-surgery. Please click here to view a larger version of this figure. 


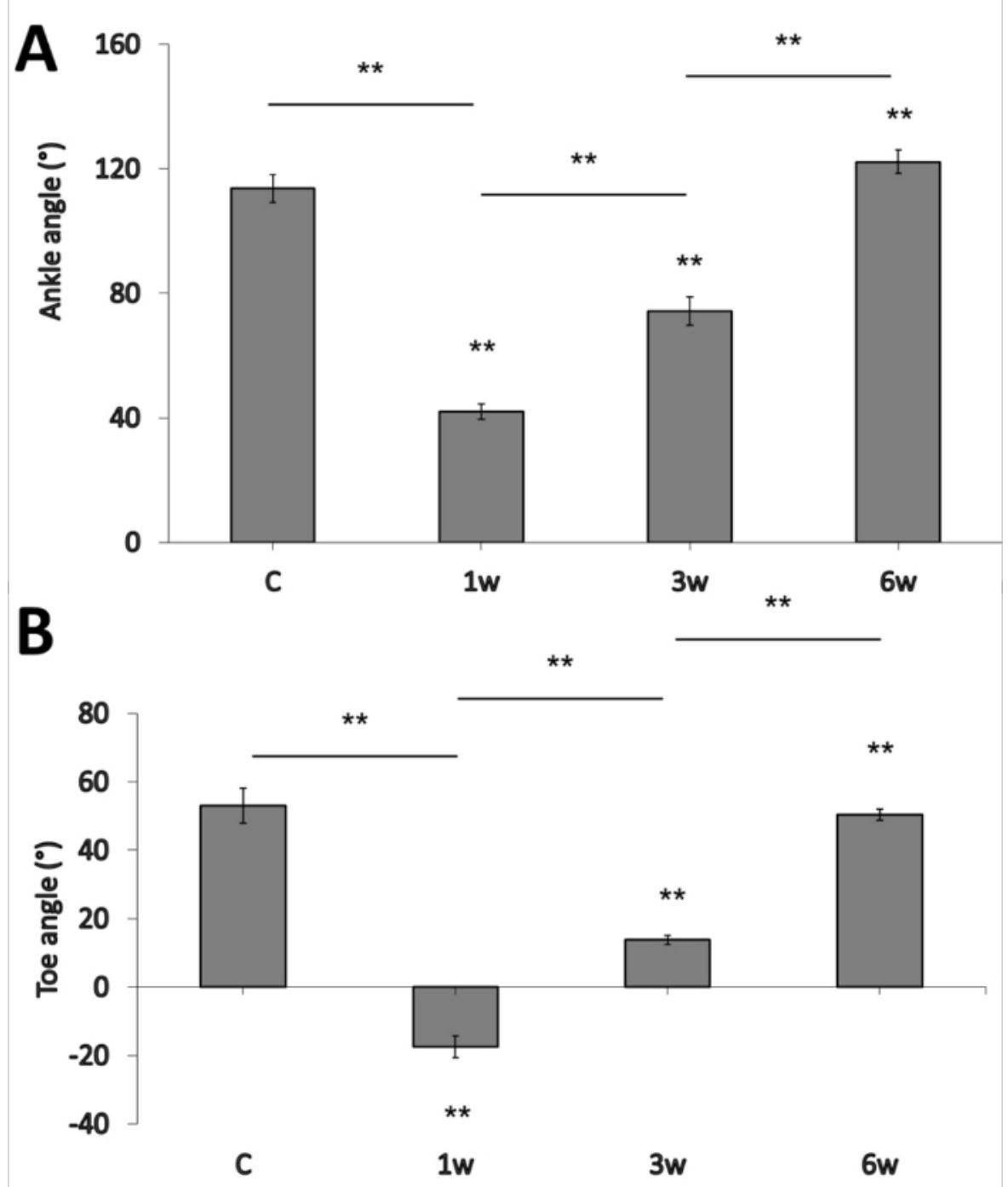

Figure 3: Ankle and toe angles. Panels A and B show changes over time in the ankle and toe angles in the 10 "toe off" phases for the control group, $1 \mathrm{w}, 3 \mathrm{w}$, and $6 \mathrm{w}$ groups $\left({ }^{* *} \mathrm{p}<0.01\right.$, compared to the control group, ${ }^{* *} \mathrm{p}<0.01$, compared to adjacent group. Error bars $=$ standard error of the mean (SEM); $C=$ control; $1 \mathrm{w}=1$ week post-surgery; $3 \mathrm{w}=3$ weeks post-surgery; $6 \mathrm{w}=6$ weeks post-surgery. Please click here to view a larger version of this figure.

Supplementary File 1. Please click here to view this file.

Supplementary File 2. Please click here to view this file.

Supplementary File 3. Please click here to view this file.

Supplementary File 4. Please click here to view this file.

Supplementary File 5. Please click here to view this file.

Supplementary File 6. Please click here to view this file.

Supplementary File 7. Please click here to view this file.

Supplementary File 8. Please click here to view this file.

Supplementary File 9. Please click here to view this file.

Supplementary File 10. Please click here to view this file.

Supplementary File 11. Please click here to view this file. 


\section{Discussion}

In this protocol, a stable and continuously walking rat is the most vital component of kinematic analysis. The treadmill speed was set to 20 $\mathrm{cm} / \mathrm{s}$. This walking speed is by no means considered "high" if rats move without space constraints ${ }^{16}$. Nevertheless, this speed is too fast for untrained rats to stably walk on the treadmill and would likely result in an abnormal gait and nonuniform movements. These events may seriously affect data reliability and authenticity. However, treadmill speeds lower than $20 \mathrm{~cm} / \mathrm{s}$ can cause the rats to intermittently stop walking, potentially producing large deviations and reduced data reliability. Therefore, training the rats to be able to steadily walk in a straight, frontward direction on the treadmill is extremely important if one is to achieve precise kinematic analysis.

Additionally, operators should not ignore the needs for reconfirmation and fine adjustments during the kinematic analysis process. We found that the swing phase accounted for $25 \%$ of the step cycle in normal rats. This means that posterior limb movements during the swing phase accelerated to the point where the camera system was not able to accurately capture the movements continually and over time. Also, excessively bright or dim ambient light, stains on the transparent treadmill sheets, and abnormal movement patterns that incidentally occur while walking could result in an exaggerated deviation of the tracing labels from the markers attached to the rats. These factors could reduce the accuracy of the motion capture process. Manual adjustments were introduced into the marker tracing system to address this issue. Using manual adjustment, evident deviations or subtle motion capture losses can be immediately rectified during the marker tracing process. Moreover, reconfirmation of changes in the curves of multiple parameters processed using the kinematic analysis software aided in searching for and correcting flaws in the marker tracing process. Reconfirmation also allowed us to generate the most reliable and authentic data.

Compared to the kinematic analysis, the shortcomings of the SFI are mainly derived from its low accuracy and reliability, rather than from interferences produced by the abovementioned factors. A previous study also noted that the SFI method is neither reliable nor reproducible when applied during the early post-injury period ${ }^{17}$. On the other hand, the high accuracy and reliability of kinematic analysis has been widely recognized. However, many previous applications were only capable of observing and measuring designated angles, especially ankle angles $^{10,15,18,19,20}$. The limitations of two-dimensional (2D) video analysis prevents investigation of additional parameters during functional evaluations.

Three-dimensional kinematic analysis overcomes all the shortcomings of SFI and enables investigation of many additional parameters. The 3D digital model is constructed from images captured by four cameras. Consequently, this apparatus can measure or calculate parameters more accurately than conventional 2D kinematic methods. Therefore, kinematic analysis that uses the 3D motion capture apparatus holds enormous promise as a potential substitute for other functional evaluation methods.

However, the 3D kinematic analysis method has several limitations. Training rodents, attaching markers, and tracing examination processes are complicated and time-consuming. In order to obtain reproducible and reliable data, the operator should be well-acquainted with the critical steps required. The skin shifting that occurs during rodent walking is particularly likely to produce data deviations ${ }^{21}$. Furthermore, the high cost of $3 \mathrm{D}$ kinematic analysis equipment may impede its popularization and limit usage in relevant studies.

Previous studies have found that the 3D kinematic analysis achieved accurate and valid results in the context of the rat sciatic nerve injury mode $^{9,22}$. Consequently, we have reasons to believe that this method may be a useful tool for functional evaluations of various trauma or disease states that involve the posterior limbs, including disorders of the central and peripheral nervous system and musculoskeletal diseases. Moreover, by altering the marker positioning, this method may be used to functionally evaluate anterior limb movements. Although these hypotheses require further verification through future experiments, we believe that kinematic analysis using a 3D motion capture apparatus may inspire more promising functional evaluation methods and play an important role in research and clinical applications.

\section{Disclosures}

The authors have nothing to disclose.

\section{Acknowledgments}

This study was supported by JSPS KAKENHI Grant Number JP19K19793, JP18H03129, and JP18K19739.

\section{References}

1. Kanaya, F., Firrell, J. C., Breidenbach, W. C. Sciatic function index, nerve conduction tests, muscle contraction, and axon morphometry as indicators of regeneration. Plastic and Reconstructive Surgery. 98 (7), 1264-1271, discussion 1272-4 (1996).

2. Takhtfooladi, M. A., Jahanbakhsh, F., Takhtfooladi, H. A., Yousefi, K., Allahverdi, A. Effect of low-level laser therapy $(685 \mathrm{~nm}, 3 \mathrm{~J} / \mathrm{cm}(2))$ on functional recovery of the sciatic nerve in rats following crushing lesion. Lasers in Medical Science. 30 (3), 1047-1052 (2015).

3. Xing, H., Zhou, M., Assinck, P., Liu, N. Electrical stimulation influences satellite cell differentiation after sciatic nerve crush injury in rats Muscle \& Nerve. 51 (3), 400-411 (2015).

4. Yang, C.C., Wang, J., Chen, S.C., Jan, Y.M., Hsieh, Y.L. Enhanced functional recovery from sciatic nerve crush injury through a combined treatment of cold-water swimming and mesenchymal stem cell transplantation. Neurological Research. 37 (90), 816-826 (2015).

5. Jiang, W. et al. Low-intensity pulsed ultrasound treatment improved the rate of autograft peripheral nerve regeneration in rat. Scientific Reports. 6, 22773 (2016).

6. Ni, X.J. et al. The Effect of Low-Intensity Ultrasound on Brain-Derived Neurotropic Factor Expression in a Rat Sciatic Nerve Crushed Injury Model. Ultrasound in Medicine \& Biology. 43 (2), 461-468 (2017). 
7. Weber, R. A., Proctor, W. H., Warner, M. R., Verheyden, C. N. Autotomy and the sciatic functional index. Microsurgery. 14 (5), $323-327$ (1993).

8. Dellon, A. L., Mackinnon, S. E. Sciatic nerve regeneration in the rat. Validity of walking track assessment in the presence of chronic contractures. Microsurgery. 10 (3), 220-225 (1989).

9. Wang, T. et al. A. Functional evaluation outcomes correlate with histomorphometric changes in the rat sciatic nerve crush injury model : A comparison between sciatic functional index and kinematic analysis. PLoS One. 13 (12), e0208985 (2018).

10. de Ruiter, G. C. et al. Two-dimensional digital video ankle motion analysis for assessment of function in the rat sciatic nerve model. Journal of the Peripheral Nervous System. 12 (3), 216-222 (2007).

11. Walker, J. L., Evans, J. M., Meade, P., Resig, P., Sisken, B. F. Gait-stance duration as a measure of injury and recovery in the rat sciatic nerve model. Journal of Neuroscience Methods. 52 (1), 47-52 (1994).

12. Dijkstra, J. R., Meek, M. F., Robinson, P. H., Gramsbergen, A. Methods to evaluate functional nerve recovery in adult rats: walking track analysis, video analysis and the withdrawal reflex. Journal of Neuroscience Methods. 96 (2), 89-96 (2000).

13. Lee, J.Y. et al. Functional evaluation in the rat sciatic nerve defect model: a comparison of the sciatic functional index, ankle angles, and isometric tetanic force. Plastic and Reconstructive Surgery. 132 (5), 1173-1180 (2013).

14. Rui, J. et al. Gait cycle analysis: parameters sensitive for functional evaluation of peripheral nerve recovery in rat hind limbs. Annals of Plastic Surgery. 73 (4), 405-411 (2014).

15. Yu, P., Matloub, H. S., Sanger, J. R., Narini, P. Gait analysis in rats with peripheral nerve injury. Muscle \& Nerve. 24 (2), $231-239$ (2001).

16. Amado, S. et al. The sensitivity of two-dimensional hindlimb joint kinematics analysis in assessing functional recovery in rats after sciatic nerve crush. Behavioural Brain Research. 225 (2), 562-573 (2011).

17. Monte-Raso, V. V., Barbieri, C. H., Mazzer, N., Yamasita, A. C., Barbieri, G. Is the Sciatic Functional Index always reliable and reproducible? Journal of Neuroscience Methods. 170 (2), 255-261 (2008).

18. Varejao, A. S. P. et al. Motion of the foot and ankle during the stance phase in rats. Muscle \& Nerve. 26 (5), $630-635$ (2002).

19. Lin, F. M., Pan, Y. C., Hom, C., Sabbahi, M., Shenaq, S. Ankle stance angle: a functional index for the evaluation of sciatic nerve recovery after complete transection. Journal of Reconstructive Microsurgery. 12 (3), 173-177 (1996).

20. Patel, M. et al. Video-gait analysis of functional recovery of nerve repaired with chitosan nerve guides. Tissue Engineering. 12 (11), 3189-3199 (2006).

21. Filipe, V. M. et al. Effect of skin movement on the analysis of hindlimb kinematics during treadmill locomotion in rats. Journal of Neuroscience Methods. 153 (1), 55-61 (2006).

22. Tajino, J. et al. Three-dimensional motion analysis for comprehensive understanding of gait characteristics after sciatic nerve lesion in rodents. Scientific Reports. 8 (1), 13585 (2018). 